\title{
Sintering Kinetics, Microstructure Development, and Graingrowth in Pure and Silica Doped Hematite*
}

\author{
By G. L. MISHRA, ${ }^{* *}$ A. R. DAS, ${ }^{* * *}$ and V.SESHADRI ${ }^{* * *}$
}

\begin{abstract}
Synopsis
Cylindrical pellets of pure and silica doped hematite were sintered at $1200^{\circ}, 1250^{\circ}, 1300^{\circ}$, and $1350^{\circ} \mathrm{C}$ for $1 / 2,1,2$, and $4 \mathrm{hr}$.

Grain size as well as variation of grain size distribution during sintering and the influence of silica additions to hematite on these parameters have been studied. Volumetric grain size distribution plots were made at various temperatures and times showing that grain growth increases with increased degree of sintering but is hindered by the presence of silica. The values of exponent $n$ in the equation $G^{n}=A t$, where $G$ is the grain diameter, $t$ is the time, and $A$ is a constant, were found to be 2.67 for pure hematite and 3.15 for $4 \%$ silica doped hematite. The calculated activation energies for grain growth were found to be 33.0 and $48.5 \mathrm{kcal} /$ mole respectively.

Porosity vs. time in $t^{(1-3 n)}$ scale where $n$ is the exponent in $G^{n}=A t$, the grain growth equation, has been plotted for pure and $4^{\circ}$ o silica doped hematite and porosity variation with time on $\log$ scale has been represented for 1,2 , and $3 \%$ silica doped hematite. The difference in porosity values from initial to final stages has been tabulated for pure and silica doped hematite. There was an increase in change of porosity with increasing silica percentage in the doped specimens. Using Coble's model of the intermediate stage sintering the variation of lattice diffusion coefficient with temperature has been plotled for pure hematite and the values are also given for $4 \%$ silica doped hematite. The activation energy for lattice diffusion was $87 \mathrm{kcal} /$ mole for pure and $115 \mathrm{kcal} /$ mole for $4 \%$ silica doped hematite respectively. Cumulative volume percent of pores vs. pore size was plotted and the slopes of the curves were found increasing with increasing degree of sintering. Volume averaged pore sizes also increased with increasing degree of sintering. The plots of percent number of pores is. asymmetry factor range have been shown for various size ranges. With increased degree of densification, the pores were observed to become more symmetrical. Photomicrographs of typical samples have been given.
\end{abstract}

\section{Introduction and Review}

The sintering mechanism has been studied extensively by many authors. ${ }^{1-8)}$ It has been generally accepted that (a) the material transport during sintering is due to the vacancy migration and (b) the driving force is due to the decrease in the free energy by the reduction of the surface area during sintering. Different models have been proposed for three stages of sintering. For the intermediate stage of sintering Coble ${ }^{5)}$ has given the diffusion models on the basis of space filling tetrakaidecahedra as the typical grain shape for bulk and grain boundary diffusion. On the basis of the bulk diffusion with grain growth model Coble has derived an equation for the rate of change in porosity with time as

$$
\frac{d P}{d t}=-\frac{10 \pi D_{v} r a_{o}^{3}}{l^{3} k T}
$$

where, $P$ : the volume fraction of pores

$t:$ the time in seconds

$D_{v}$ : the volume diffusion crefficients for material transport

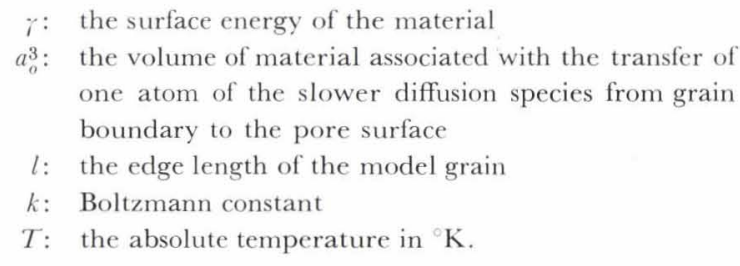

Besides, $l$ is related to $X$, the average linear intercept of microscopic count ${ }^{8)}$ as

$$
l=0.6 X
$$

and $X$ is related to the true average grain size ${ }^{9)}$ as

$$
G=\frac{\pi}{2} X
$$

Combining Eqs. 1 to 3

$$
\frac{d P}{d t}=\frac{-10 \pi(\pi / 2)^{3} D_{v \gamma} a_{o}^{3}}{(0.6)^{3} G^{3} k T}
$$

Grain size varies with time during sintering ${ }^{10)}$ and it is given as

$$
G^{n}=A t
$$

where $n$ is a constant and $A$ is given as

$$
A=A_{o} \exp \left(Q_{g} / R T\right)
$$

where $Q_{\text {q }}$ is the activation energy for grain growth. Substituting Eq. (5) in Eq. (4)

$$
\frac{d P}{d t}=\frac{-10 \pi(\pi / 2)^{3} D_{v} \gamma a_{o}^{3}}{(0.6)^{3} k T A^{3 / n}} t^{-3 / n}
$$

Integration of Eq. (7) for $n \neq 3$ gives

$$
P=J(T)\left[t_{f}^{(1-3 / n)}-t^{(1-3 / n)}\right]
$$

where $t_{f}$ is the time for sintering where final porosity becomes zero and

$$
J(T)=\frac{563 D_{v} a_{o}^{3} \gamma}{(1-3 / n) k T A^{3 / n}}
$$

Plot of $P$ vs. $t^{(1-3 / n)}$ gives a straight line, from the slope of which $D_{v}$ can be calculated.

In general $D_{v}$ is of the form

$$
D_{v}=D_{o} \exp \left(-Q_{D} \mid R T\right)
$$

Then from Eqs. (6) and (10) we can rewrite Eq. (9) as

$$
J(T)=\frac{563 D_{o} a_{o \gamma}^{3}}{(1-3 / n) A_{o} k T} \exp -\left(\frac{Q_{D}-3 / n Q_{g}}{R T}\right) \ldots
$$

* Received May 19, 1970.

** Post graduate student, Indian Institute of Technology Kanpur, U.P., India.

*** Department of Metallurgical Engineering, Indian Institute of Technology Kanpur, U.P., India. 
The term $Q_{D}-3 / n Q_{g}=Q_{P}$ may be defined as the activation energy for the process. Since the activation energy for lattice diffusion $Q_{D}$ may be assumed constant for a material, the value of $Q_{P}$ may be either positive or negative depending on whether $3 / n Q_{g}$ is less than or greater than $Q_{D}$.

Less attention has however been paid to the characterization of pores during the sintering process. The shape, size as well as the distribution of the pores have remarkable effect on the sintering characteristics. For a fixed porosity, fine and well distributed pores lead to uniform sintering and higher final densities before grain growth offers impediments to pore removal. In a real material, many pores of different sizes exist because of non-uniform particle packing and bridging between the particle aggregates. Francois and Kingery ${ }^{11)}$ have discussed the requirements for pore stability and pointed out the conditions for growth or shrinkage in terms of the number of sides of a pore and the dihedral angles between the grain boundary and the pore sides.

There will also be a tendency for material transfer from the grain boundary towards both large and small pores but large pore shrink more slowly. Francois and Kingery have said that the important factor is not pore size but the ratio of pore size to grain size. Largest diameter pores grow and this growth continues until there has been sufficient grain growth to increase the grain size relative to the pore size.

Kingery and Francois ${ }^{22}$ also showed that if the rate of grain growth is much faster than the rate of pore removal, then the value of $n$ in the grain growth equation $G^{n}=A t$ could be 3 , as used in Coble's ${ }^{5)}$ model. However in the case of many oxides different values have been reported ${ }^{13}$ ) for different oxides.

Comparatively the studies on the sintering of mixed oxides have been less extensive. ${ }^{11), 12)}$ It was shown that the interfacial energy plays a very important role in the sintering of mixed oxides.

In the present work the sintering kinetics of pure and $\mathrm{SiO}_{2}$ doped hematite has been studied. The effect of $\mathrm{SiO}_{2}$ on the densification and grain growth has been observed. By microscopic examinations and pore size measurements the contribution of different size pores toward the densification has been estimated. From grain size measurements the distribution of grains for different degrees of sintering has been determined.

\section{Experimental Method}

Analar grade hematite powder of $99.4 \%$ in purity and of size -325 mesh was used for the studies. Densification studies were carried out both on pure and $\mathrm{SiO}_{2}$ doped hematite while pore characterization was conducted on the former. In order to make the starting powder characteristics similar in both experiments, the pure material was subjected to presintering at $1350{ }^{\circ} \mathrm{C}$ for $4 \mathrm{hr}$ in oxygen atmosphere in the form of $1 / 2^{\prime \prime}$ dia. cylindrical pellet pressed at $5000 \mathrm{lbs}$ load with $6 \%$ dextrine plus $6 \%$ moisture as binder.

After sintering, the pellets were crushed in a mortar up to -20 mesh and subsequently ground with water in a jar mill for $22 \mathrm{hr}$ and reduced to -325 mesh powder. The material was filtered and dried.

Final pellets of $1 / 2^{\prime \prime}$ diameter were similarly pressed by taking $2 \mathrm{~g}$ of material each. The pellets were preheated at $800^{\circ} \mathrm{C}$ for $2 \mathrm{hr}$ in oxygen atmosphere to remove dextrine and moisture and provide strength to withstand cracking during rapid introduction into high temperature during isothermal sintering. Sintering was done at temperatures of $1200^{\circ}, 1250^{\circ}$, $1300^{\circ}$, and $1350^{\circ} \mathrm{C}$ for $1 / 2,1,2,3$, and $4 \mathrm{hr}$ in a horizontal silicon carbide heated tube furnace in conjunction with electromax on-off temperature controller. The temperature was controlled to $\pm 5^{\circ} \mathrm{C}$ and an uniform zone of $8 \mathrm{~cm}$ at the centre was available.

Weight, diameter, and length of each pellet was measured before and after sintering. True density of the powder was measured by specific gravity bottle.

Porosity $P$ was calculated as $P=\left(\pi r^{2} h-M / d_{o}\right) /\left(M / d_{o}\right)$ where $r, h, M$ are the radius, length, and the weight of the pellet respectively, and $d_{o}$ is the true density of powder.

For pore characteristics, the pellets sintered for $1 / 2$, 2, and $4 \mathrm{hr}$ at $1250^{\circ}, 1300^{\circ}$, and $1350^{\circ} \mathrm{C}$, were examined. Each pellet, after mounting in a plastic, was polished through emery paper and then on 0.5 micron alumina powder using a syntron vibratory polisher for $40 \mathrm{hr}$.

Pore size was measured on a metallograph with a movable and rotating stage. Measurements were done along a line without missing any visible pore on $400 X$ magnification. For small pores of simple shapes the maximum length and maximum breadth of the pores were recorded. The pores which were long and complicated (vide Photo. 1 (p. 225)) were divided into several convenient parts of lengths $y_{1}, y_{2}, y_{3}$ and maximum widths, $b_{1}, b_{2}$, and $b_{3}$ respectively. The volume of the pore or part of it, was determined by assuming it to be a cylinder of radius equal to the breadth of the pore. The total volume of the pore is given by

$$
V=\frac{\pi b_{1}^{2}}{4} y_{1}+\frac{\pi b_{2}^{2}}{4} y_{2}+\frac{\pi b_{3}^{2}}{4} y_{3} \text {. }
$$

Now this volume was equated to the volume of a sphere of diameter $X$. One hundred measurements were taken for each sample. Volume averaged pore size was calculated by the equation.

$$
\bar{X}=\frac{\sum x_{i} V_{i}}{V}
$$

where $\bar{X}$ : the volume averaged pore size

$V_{i}$ : the volume of pores in certain size ranges

$x_{i}:$ the logrithmic average size of that range

$V$ : the total volume of pores.

The above procedure admittedly neglects to consider the true distribution of the pores which are different from the apparent distribution on polished planes, ${ }^{14}$ ) but is simpler, and, as will be seen, does not detract from the results and conclusions that follow.

Asymmetry factor was defined as equal to $y / b$ where $y$ is the length and $b$ is the breadth of the pore. It 

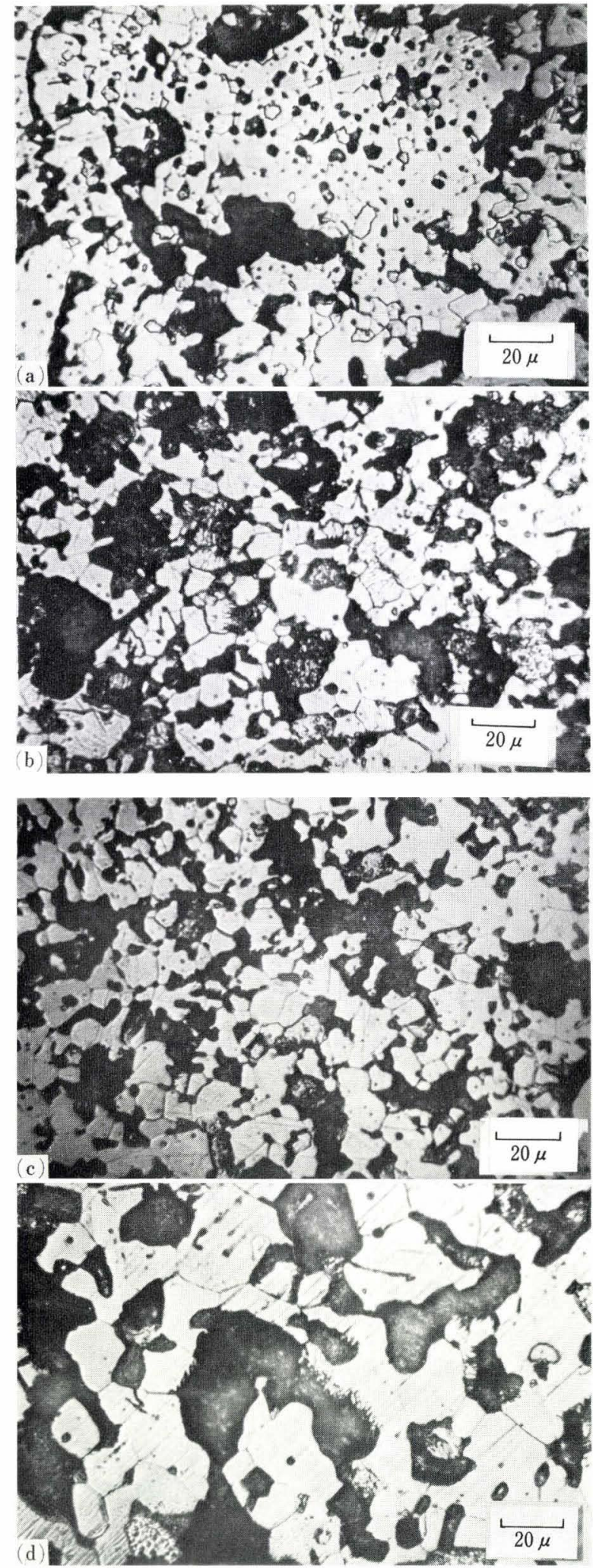

Photo. 1. Photomicrographs of a etched hematite pellets showing grains
(a) $1250^{\circ} \mathrm{C}, 1 \mathrm{hr}$
(b) $1300^{\circ} \mathrm{C}, 1 \mathrm{hr}$
(c) $1300^{\circ} \mathrm{C}, 3 \mathrm{hr}$
(d) $1350^{\circ} \mathrm{C}, 4 \mathrm{hr}$

is a measure of sphericity so that for a sphere asymmetry factor is unity.

Hematite pellets sintered at $1250^{\circ}, 1300^{\circ}, 1350^{\circ} \mathrm{C}$ for $1 / 2,1,2,3$, and $4 \mathrm{hr}$ were polished for grain growth studies. The pellets were etched in boiling hydrochloric acid for 2 min. Marten's diameter of the grains were measured along a line. Silica doped hematite pellets were similarly treated. Grain size measurements were taken on one hundred points on each sample. The volume averaged grain diameter was determined by the formula

$$
X=\left\{\begin{array}{c}
\sum n_{i} x_{i}^{3} \\
\sum n_{i}
\end{array}\right\}^{1 / 3}
$$

where $X$ is the volume averaged grain size and $n_{i}$ is the number of grains with diameter $x_{i}$. The true average grain diameter was found by Fullman's ${ }^{9)}$ relation.

$$
G=\frac{\pi}{2} X
$$

where $G$ is the true average grain diameter.

$\mathrm{X}$-ray diffraction patterns were taken for pure hematite and 4\% silica doped hematite. No additional lines were observed for silica doped hematite. Microstructure observation for the presence of second phase was inconclusive because polished and etched samples of both pure and silica doped pellets shows small regions of contrast. This was probably attributable to the orientation effects of some grains which etch more easily than others.

\section{Results and Discussion}

\section{Grain Growth}

The cumulative volumetric distribution plots of grains are shown in Figs. 1 (a), (b), and (c) at temperatures $1250^{\circ}, 1300^{\circ}, 1350^{\circ} \mathrm{C}$ and for various times, while Fig. 2 gives the same plot for the doped hematite at $1250^{\circ}$ and $1350^{\circ} \mathrm{C}$ for different times. In all these cases the curves are shifted to the right with increasing degree of sintering, indicating the grain growth with increasing degree of sintering. The upper portions of these curves approach a limiting size signifying that the largest grains are growing very slowly.

In the distribution plots, the average slopes of near linear regions indicate the degree of uniformity. The slopes of the near linear regions of these curves tend to increase with increased degree of sintering except in a few cases showing that the size distribution is becoming more uniform. If the smaller grains are growing faster than the larger ones, the slope will increase and the distribution should become more uniform.

Average grain size $G$ is increasing with increased degree of sintering in the case of pure as well as silica doped hematite samples as shown in Fig. 3 and Table 1 respectively. The dependence of grain size on sintering time has been given as $G^{n}-G_{o}^{n}=A t$, which reduces to $G^{n}=A t$ when $G_{o}^{n} \ll G^{n}$.

In this case $n$ is a constant and $A$ is given as $A=$ $A_{o} e^{-Q_{g} / R T}$. Here $A_{0}$ is the pre-exponential factor and $Q_{g}$ is the activation energy for grain growth. Plot of $\log G$ vs. $\log t$ gives the slope equal to $1 / n$ and the 


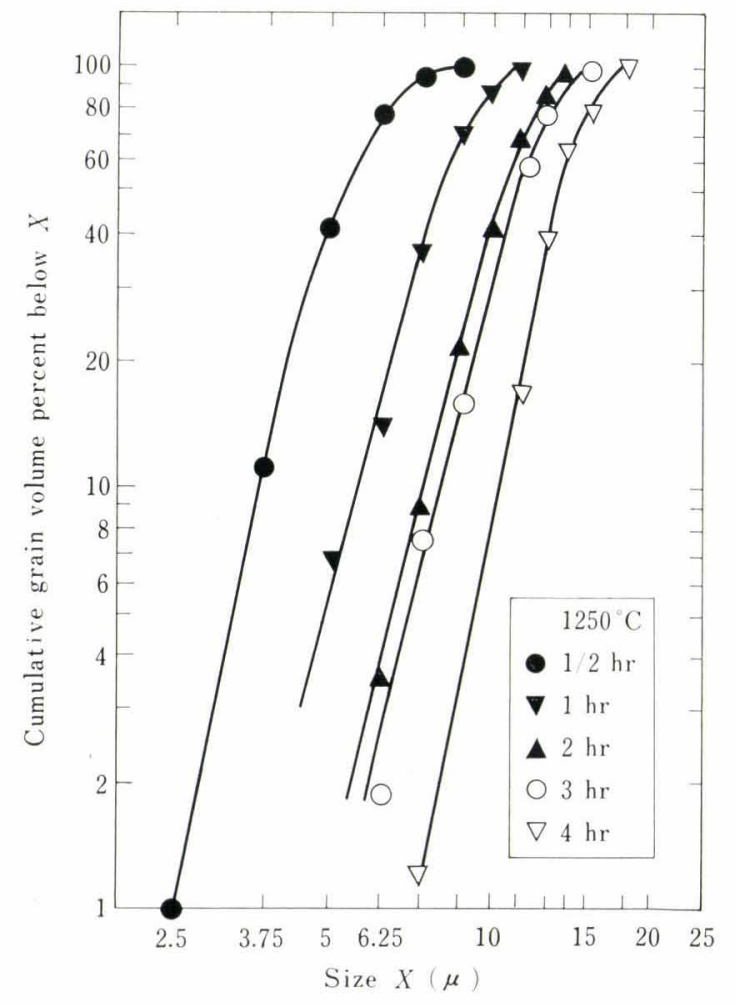

(a)

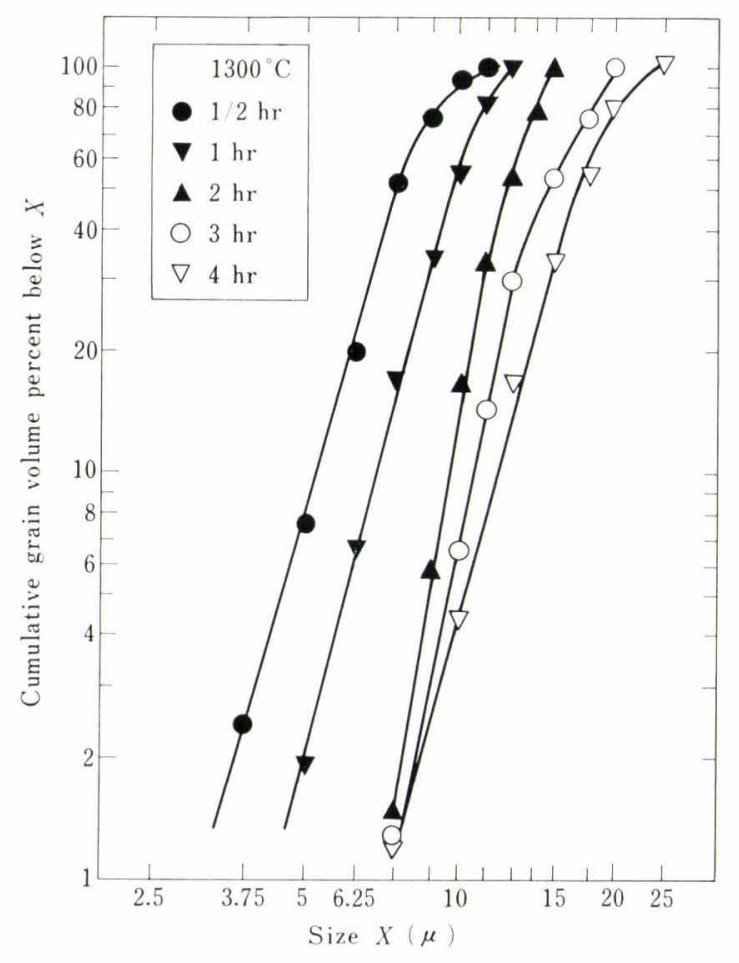

(b)

Fig. 1. Grain size distribution in pellets of pure hematite
(a) $1250^{\circ} \mathrm{C}$
(b) $1300^{\circ} \mathrm{C}$
(c) $1350^{\circ} \mathrm{C}$

(Right above)

intercept equal to $\log A / n$. From the slope of the plot of $\log A$ vs. 1/T, $Q_{g}$ can be determined.

The value of $n$ was 2.67 in the case of pure hematite and 3.15 in the case of $4 \%$ silica doped hematite. Similar values have been reported for oxides such as

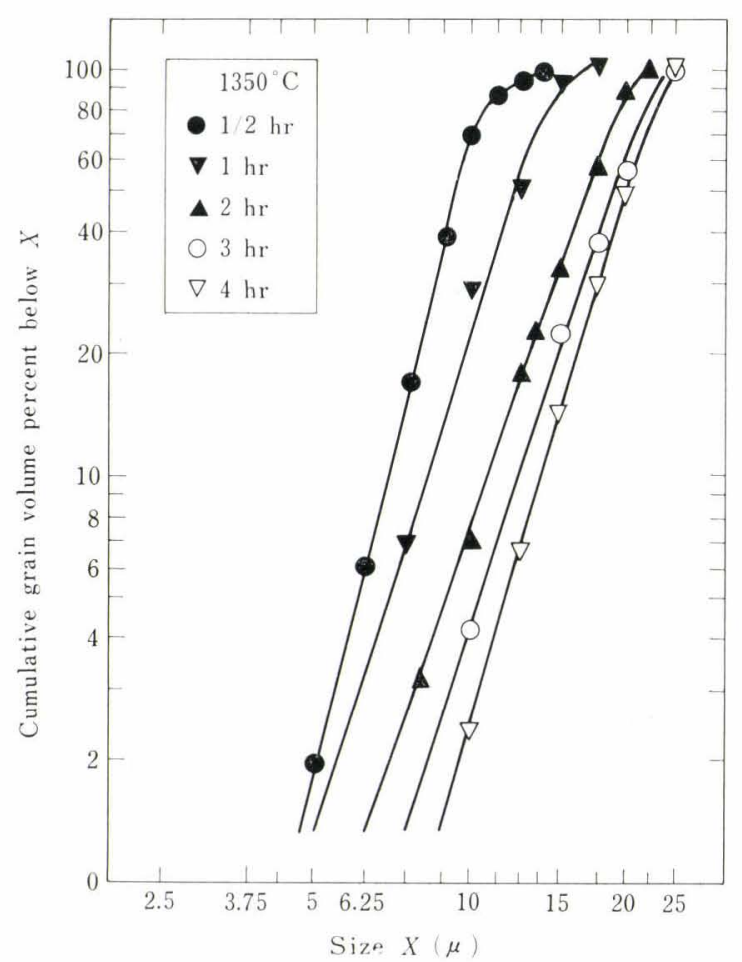

(c)

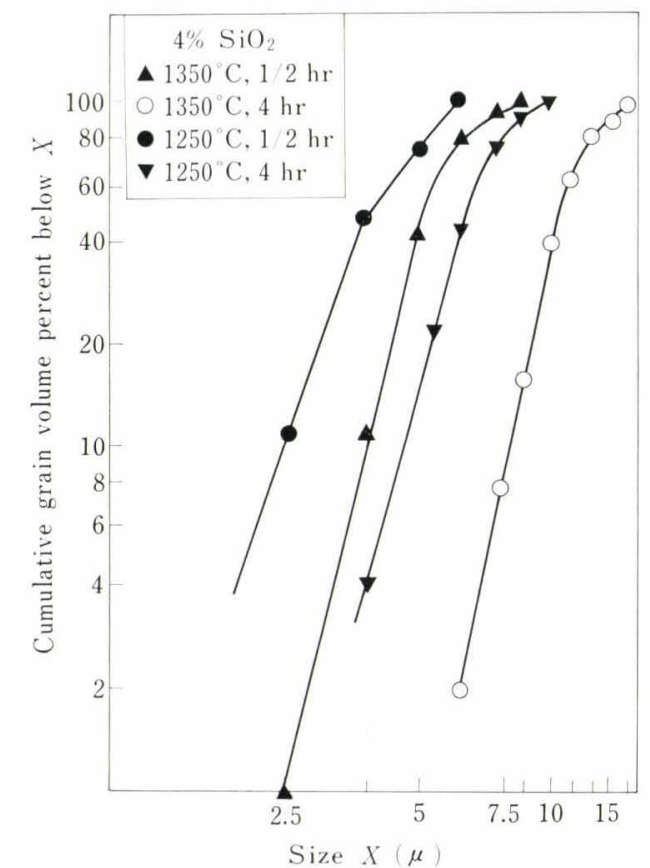

Fig. 2. Grain size distribution in pellets of hematite with $4 \% \mathrm{SiO}_{2}$

3 for $\mathrm{Al}_{2} \mathrm{O}_{3}, 2$ for $\mathrm{MgO}$ and $\mathrm{CaO}, 2$ for $\mathrm{BeO}$ and 2.5 for $\mathrm{Ca}_{0.16} \mathrm{Zn}_{0.84} \mathrm{O}_{1.84} \cdot{ }^{13)}$

When the value of $n$ is different from 3 the diffusion equation given by Coble ${ }^{5)}$ is modified as in Eq. (8).

The activation energy for grain growth is $33 \mathrm{kcal}$ mole for pure hematite as calculated from Fig. 4 and $48.5 \mathrm{kcal} / \mathrm{mole}$ in the case of $4 \% \mathrm{SiO}_{2}$ doped hematite as shown in Table 1 . The value is higher in the case of doped specimens due probably to the presence of $\mathrm{SiO}_{2}$ which should be present as a second phase ${ }^{15)}$ and will hinder the grain growth. When a grain boundary 


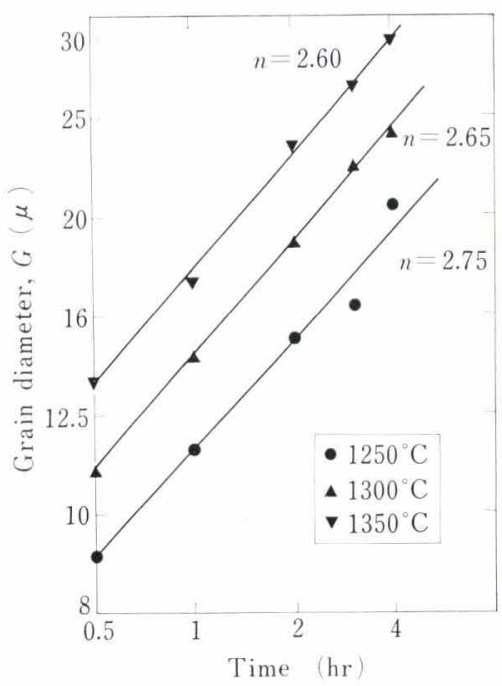

Fig. 3. Variation of grain size with time for pure hematite pellets

Table 1. Values of average grain size, $n, \log A$, and $Q_{n}$ for $4 \% \mathrm{SiO}_{2}$ doped hematite

\begin{tabular}{ccrccc}
$\begin{array}{c}\text { Temp. } \\
\left({ }^{\circ} \mathrm{C}\right)\end{array}$ & $\begin{array}{c}\text { Time } \\
(\mathrm{hr})\end{array}$ & $\begin{array}{c}\text { Average } \\
\text { grain size } \\
(/ \mathrm{l})\end{array}$ & $n$ & $\log A$ & $\begin{array}{c}Q_{g}^{\prime \prime} \\
(\mathrm{kcal} / \mathrm{mole})\end{array}$ \\
\hline 1250 & $1 / 2$ & 5.5 & 3.2 & 2.7 & \\
\hline \multirow{2}{*}{1350} & 4 & 10.4 & & & 48.5 \\
\cline { 1 - 1 } & $1 / 2$ & 8.1 & 3.1 & 3.1 & \\
& 4 & 15.5 & & &
\end{tabular}

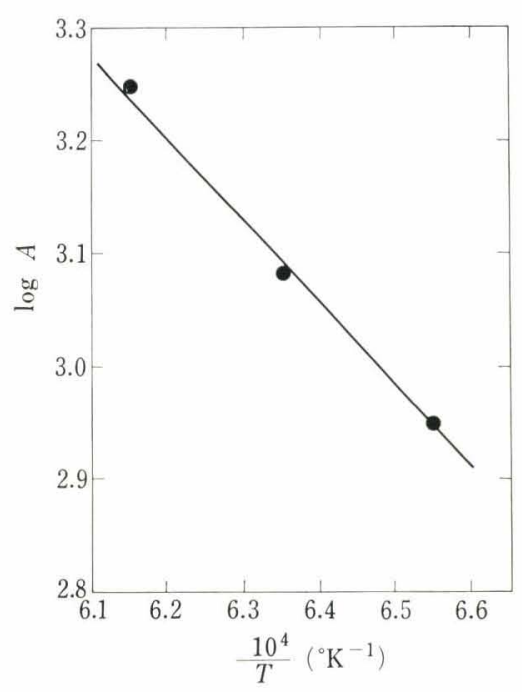

Fig. 4. Plot of $\log A$ vs. 1/T

passes through the dispersed phase then either it has to cut through the silica particles or it has to carry them along. The energies required for both these processes are higher than the case when no second phase. Therefore the activation energy for grain growth in the case of $\mathrm{SiO}_{2}$ doped hematite would be more than the pure hematite.

\section{Sintering Kinetics}

The slopes of the lines in Figs. 5 (a), (b), and 6 (a),

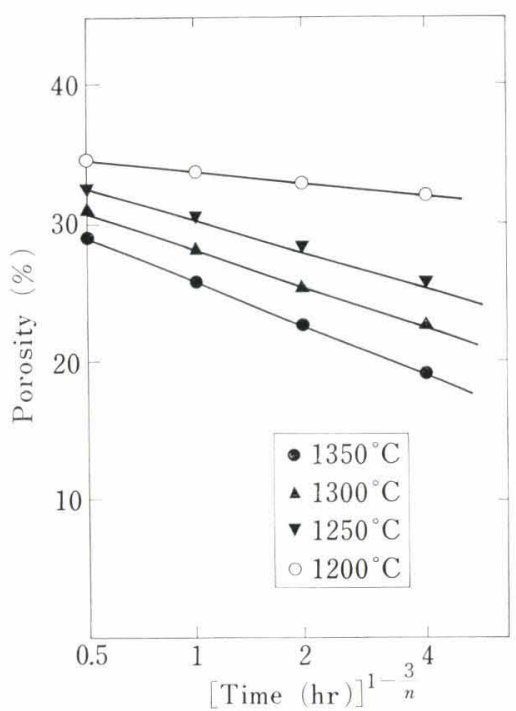

(a)

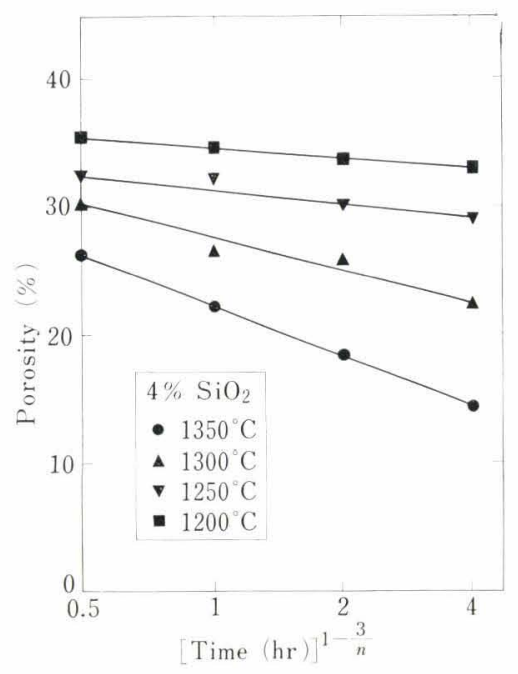

(b)

Fig. 5. Plot of porosity vs. time

(a) Pure hematite

(b) $4 \% \mathrm{SiO}_{2}$ doped hematite

(b), (c) are observed to increase with temperature indicating a positive value of $Q_{P}$ in Eq. (11). For pure and $4 \%$ silica doped hematite the porosity has been plotted with (time) $)^{1-3 / n}$ in Figs. 5 (a) and (b) where $n$ is the exponent in the equation $G^{n}=A t$. The value of $n$ has been calculated for pure and $4 \%$ silica doped hematite as 2.67 and 3.15 respectively. The straight lines were fitted as predicted by Eq. (8).

For 1, 2, and 3\% silica doped hematite, porosity vs, log time was plotted in Figs. 6 (a), (b), and (c) because the values of $n$ were not available to use Eq. (8). Near straight lines were observed, which is due probably to the values of $n$ being very close to 3 , aud Coble's model predicts this linear relationship when $n=3$.

The lattice diffusion coefficient $D_{v}$ of slow moving ion $\mathrm{Fe}^{3+}$ has been calculated from Eq. (9) by taking the values of surface energy as $1000 \mathrm{ergs} / \mathrm{cm}^{2}$. For hematite, $a_{0}^{3}$ has been calculated as 


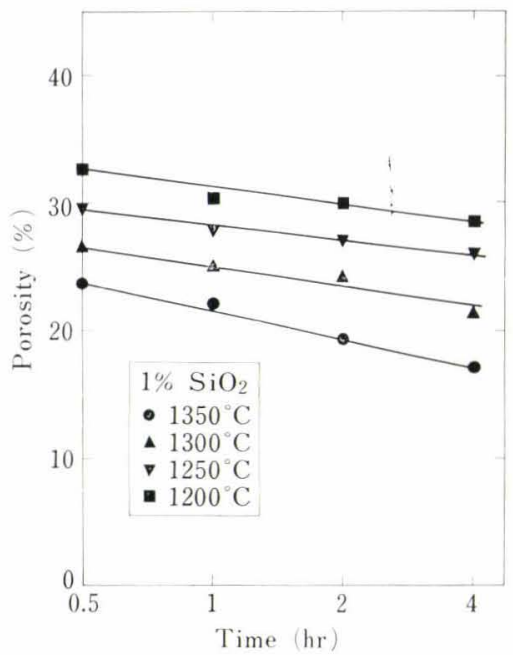

(a)

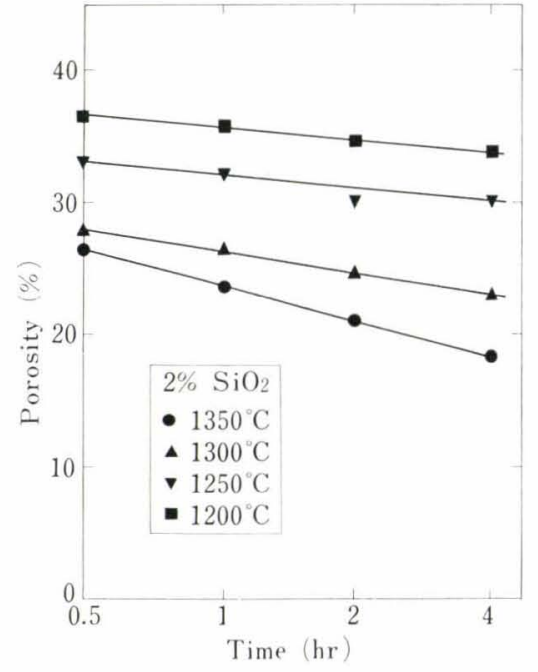

(b)

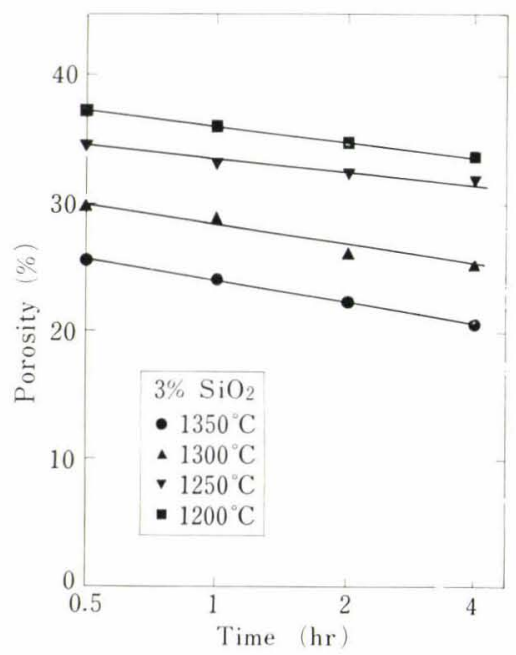

(c)

Fig. 6. Plot of porosity vs, time in $\log$ scale
(a) $1 \% \mathrm{SiO}_{2}$
(b) $2 \% \mathrm{SiO}_{2}$
(c) $3 \% \mathrm{SiO}_{2}$ doped hematite

$$
a_{o}^{3}=\frac{\text { Molecular weight }}{2 d N_{o}}=2.6 \times 10^{-23} \mathrm{~cm}^{3}
$$

where $d$ is the density of the material and $\mathcal{N}_{o}$ is Avogadro's number.

$\log D_{v}$ vs. $1 / T$ plot is shown in Fig. 7 for pure hematite. A reasonably good straight line is observed which is close to the values of Himmel, Mehl, and Birchenal ${ }^{16)}$ but deviates from Coble's data ${ }^{2)}$ for initial state sintering of pure hematite. The values of lattice diffusion coefficient for $4 \%$ silica doped hematite were calculated as $2.0 \times 10^{-9} \mathrm{~cm}^{2} / \mathrm{sec}$ at $1350^{\circ} \mathrm{C}$ and $1.95 \times 10^{-10} \mathrm{~cm}^{2} / \mathrm{sec}$ at $1250^{\circ} \mathrm{C}$ which may be compared with the values of pure hematite in Fig. 7 .

The activation energies $Q_{p}$ for the process calculated from Eq. (11) were $50 \mathrm{kcal} /$ mole and $69 \mathrm{kcal} / \mathrm{mole}$ for pure and $4 \%$ silica doped hematite respectively. The activation energies $Q_{\text {g }}$ for grain growth for the two compositions were also determined as $33 \mathrm{kcal} / \mathrm{mole}$ and $48.5 \mathrm{kcal} / \mathrm{mole}$ respectively. Consequently the activation energy $Q_{D}$ for lattice diffusion which is given by $Q_{D}=Q_{p}+3 / n Q_{g}$ was calculated to be $87 \mathrm{kcal} /$ mole for pure hematite and $115 \mathrm{kcal} / \mathrm{mole}$ for the silica doped hematite.

The differences in porosities $\Delta p$ of initial and final states are shown in Table 2 for various times and temperatures of sintering. From Eq. (8) we can write

$$
\Delta p=\left(p_{o}-p\right)=\frac{563 D_{v} a_{o}^{3} \gamma}{k T(1-3 / n) A^{3 / n}} t^{(1-3 / n)}
$$

It can be noted that the ratio of $D_{v} / A^{3 / n}$ increases with silica content and as a consequence $J P$, the change in porosity showed increase with silica percentage. This effect is more pronounced at higher temperatures particularly at $1350^{\circ} \mathrm{C}$ but at lower temperatures there was insufficient sintering resulting in a scatter of data.

It will be noticed from Table 2 that the results of pure hematite does not completely fall in line with those of the doped material. Although attempt was

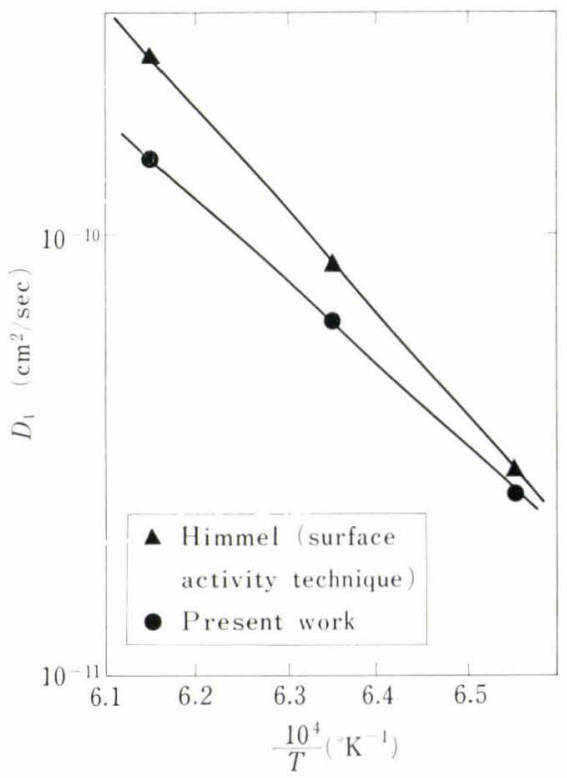

Fig. 7. Plot of $\log D_{v}$ vs. 1/T

made to make the initial powder characteristics of both pure and doped hematite similar by presintering, this difference may be attributable to initial powder differences.

From the phase diagram of $\left.\mathrm{FeO}-\mathrm{Fe}_{2} \mathrm{O}_{3}-\mathrm{SiO}_{2}{ }^{15}\right)$ system no compound formation could be inferred within the ranges of these silica composition. From X-ray patterns for pure and 4\% silica doped hematite no difference could be observed. True density of the powder was determined by specific gravity bottle and the density values were 5.093, 4.981, 4.950, 4.90, $4.851 \mathrm{~g} / \mathrm{cm}^{3}$ for pure hematite and 1, 2, 3, 4\% silica doped hematite respectively. These values were nearly equal to the theoretical density which is given by

True density $=(\%$ hematite $\times$ density of hematite $+\% \mathrm{SiO}_{2} \times$ density of $\left.\mathrm{SiO}_{2}\right) / 100$ 


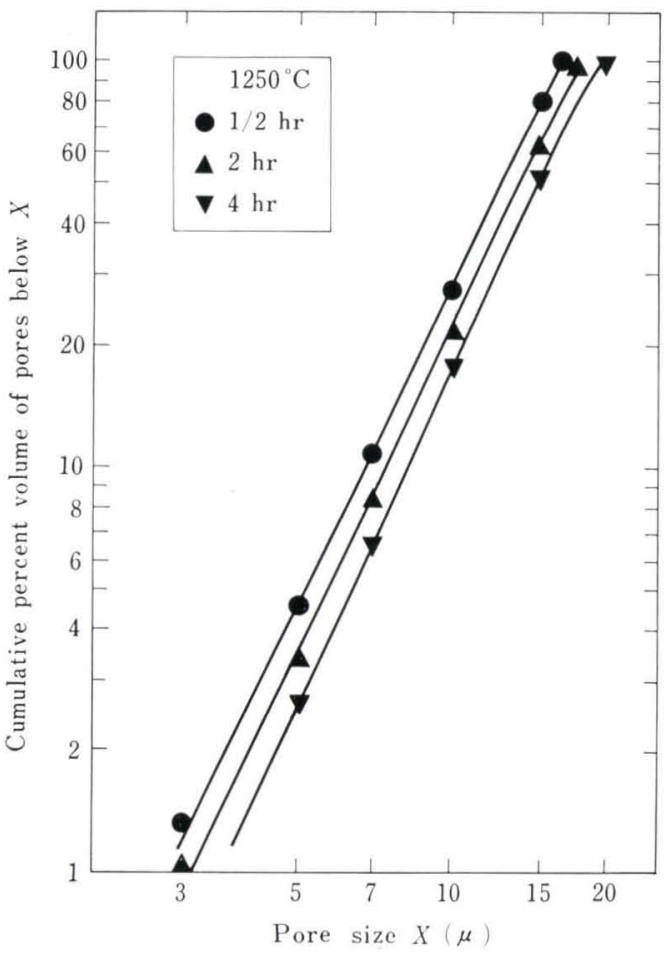

(a)

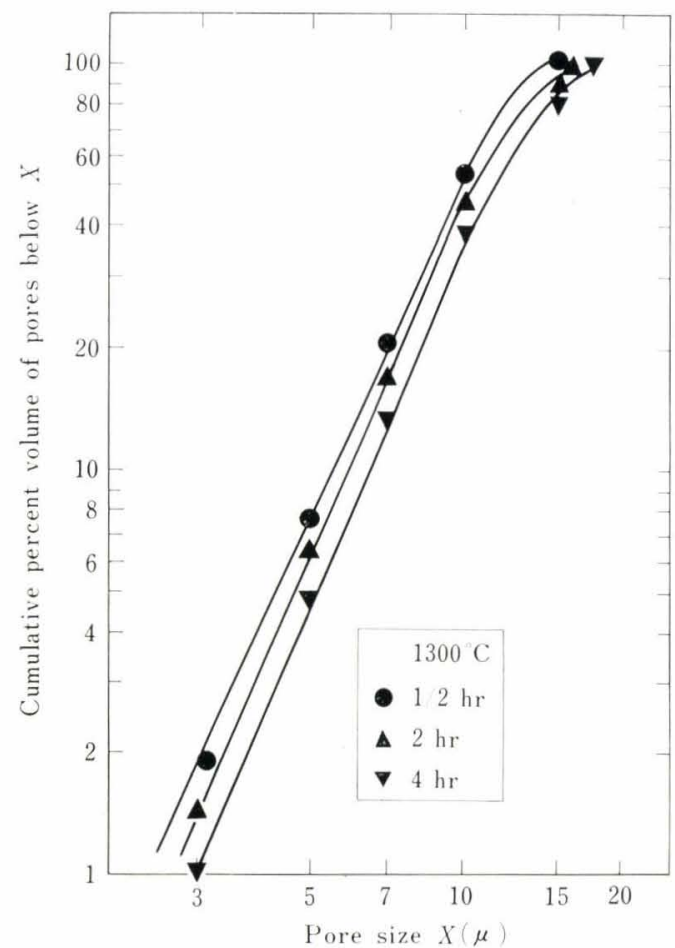

(b)

Fig. 8. Distribution of pores in hematite pellets sintered at
(a) $1250^{\circ} \mathrm{C}$
(b) $1300^{\circ} \mathrm{C}$
(c) $1350^{\circ} \mathrm{C}$

(Right above)

Cumulative volume percent below size $X$ vs. size $X$ is shown in Figs. 8 (a), (b), and (c) for sintering temperatures of $1250^{\circ}, 1300^{\circ}$, and $1350^{\circ} \mathrm{C}$ respectively. Curves are reasonably linear except the upper portion which approaches a limiting size. Slopes of the curves increase from $1250^{\circ}$ to $1300^{\circ} \mathrm{C}$ and decrease from $1300^{\circ}$ to $1350^{\circ} \mathrm{C}$. The slope also increases with in-

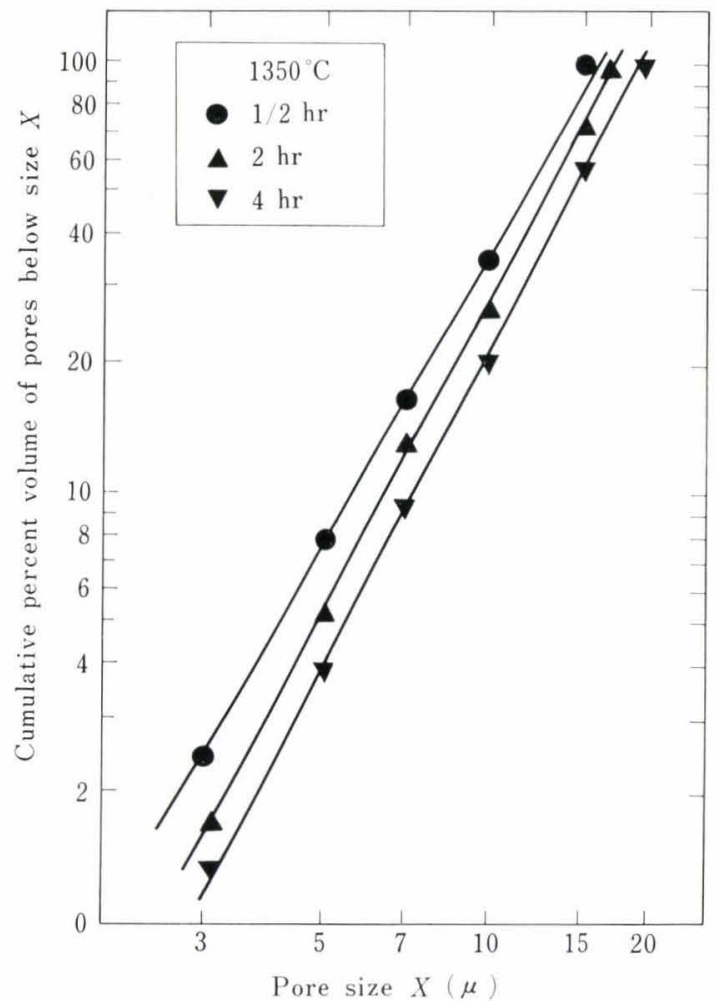

(c)

Table 2. Values of porosity change $\Delta P$ for different silica percentages

\begin{tabular}{|c|c|c|c|c|c|c|}
\hline \multirow{2}{*}{$\begin{array}{l}\text { Temp. } \\
\left({ }^{\circ} \mathrm{C}\right)\end{array}$} & \multirow{2}{*}{$\begin{array}{l}\text { Time } \\
\text { (hr) }\end{array}$} & \multicolumn{5}{|c|}{$\Delta P$} \\
\hline & & $\begin{array}{c}\text { Pure } \\
\text { hematite }\end{array}$ & $\begin{array}{l}1 \% \\
\mathrm{SiO}_{2}\end{array}$ & $\begin{array}{l}2 \% \\
\mathrm{SiO}_{2}\end{array}$ & $\begin{array}{l}3 \% \\
\mathrm{SiO}_{2}\end{array}$ & $\begin{array}{l}4 \% \\
\mathrm{SiO}_{2}\end{array}$ \\
\hline \multirow{4}{*}{1350} & $1 / 2$ & 16.0 & 15.5 & 16.2 & 17.8 & 15.6 \\
\hline & 1 & 17.3 & 16.7 & 18.7 & 19.0 & 22.2 \\
\hline & 2 & 19.7 & 18.2 & 20.6 & 20.7 & 24.1 \\
\hline & 4 & 24.2 & 21.5 & 22.1 & 23.0 & 28.7 \\
\hline \multirow{4}{*}{1300} & $1 / 2$ & 12.3 & 11.9 & 14.2 & 13.4 & 12.2 \\
\hline & 1 & 14.8 & 12.7 & 14.4 & 14.4 & 15.5 \\
\hline & 2 & 16.7 & 13.9 & 16.7 & 17.0 & 16.2 \\
\hline & 4 & 20.5 & 17.1 & 17.9 & 17.7 & 19.8 \\
\hline \multirow{4}{*}{1250} & $1 / 2$ & 11.4 & 9.1 & 8.2 & 8.9 & 9.8 \\
\hline & 1 & 12.7 & 10.3 & 9.5 & 9.8 & 9.8 \\
\hline & 2 & 14.5 & 11.5 & 11.7 & 10.6 & 12.6 \\
\hline & 4 & 18.0 & 12.2 & 13.0 & 10.9 & 13.4 \\
\hline \multirow{4}{*}{1200} & $1 / 2$ & 9.5 & 6.0 & 6.0 & 6.1 & 7.0 \\
\hline & 1 & 10.0 & 7.8 & 6.0 & 7.6 & 7.6 \\
\hline & 2 & 10.8 & 8.3 & 7.5 & 8.5 & 8.9 \\
\hline & 4 & 11.3 & 10.0 & 9.1 & 9.9 & 9.4 \\
\hline \multicolumn{2}{|c|}{$\begin{array}{l}\text { Initial } \\
\text { porosities }\end{array}$} & 42.5 & 38.8 & 41.8 & 43.4 & 42.3 \\
\hline
\end{tabular}

creasing time at $1250^{\circ}$ and $1300^{\circ} \mathrm{C}$, while it decreases at $1350^{\circ} \mathrm{C}$ as shown in Table 3. Finer pores are expected to shrink and disappear at a faster rate than the coarser ones. This will result in the distribution becoming narrower and an increase in the slope of Fig. 8. 


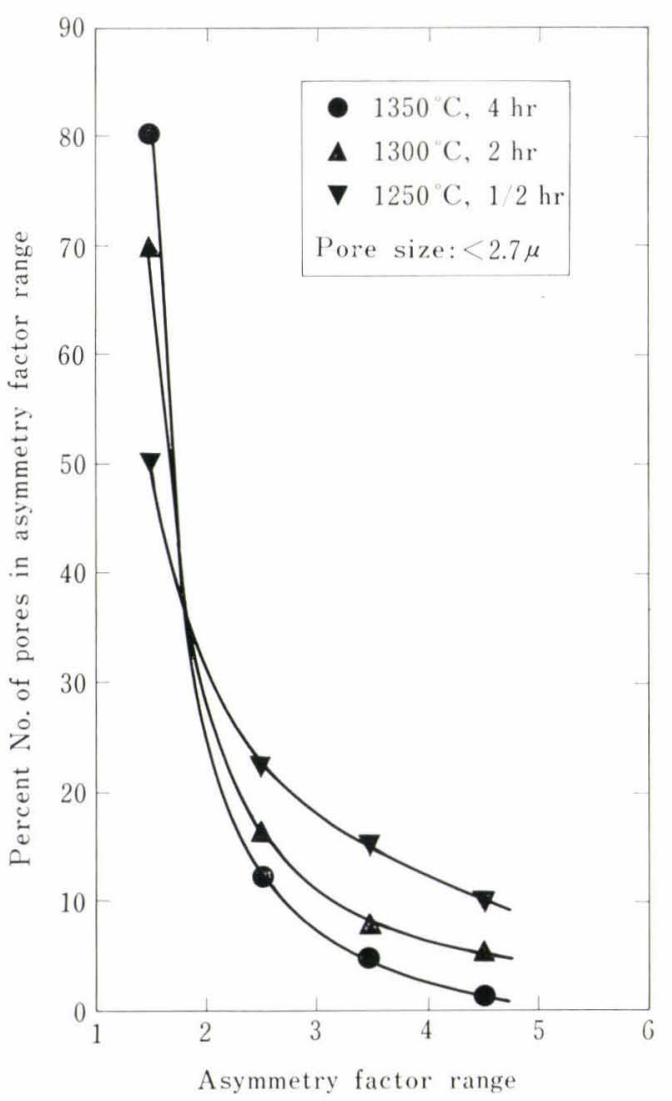

(a)

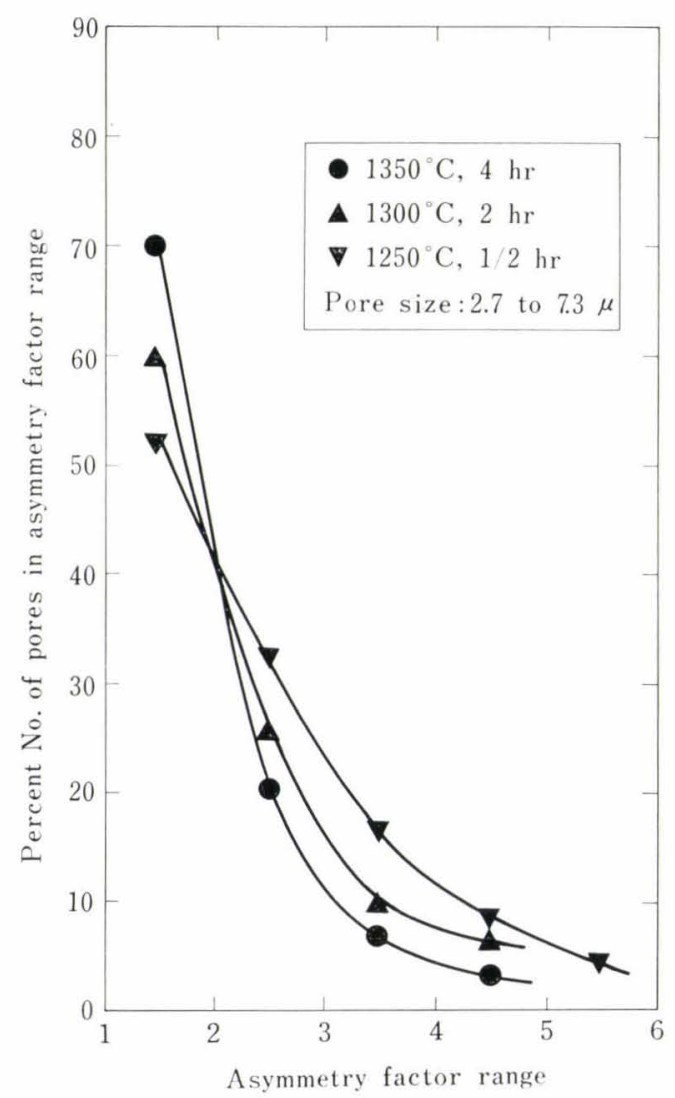

(b)

Fig. 9. Asymmetry characteristics of pores in sintered hematite pellets size ranges
(a) $<2.7 \mu$
(b) For 2.7 to $7.3 \mu$
(c) $>7.3 \%$ (Right above)

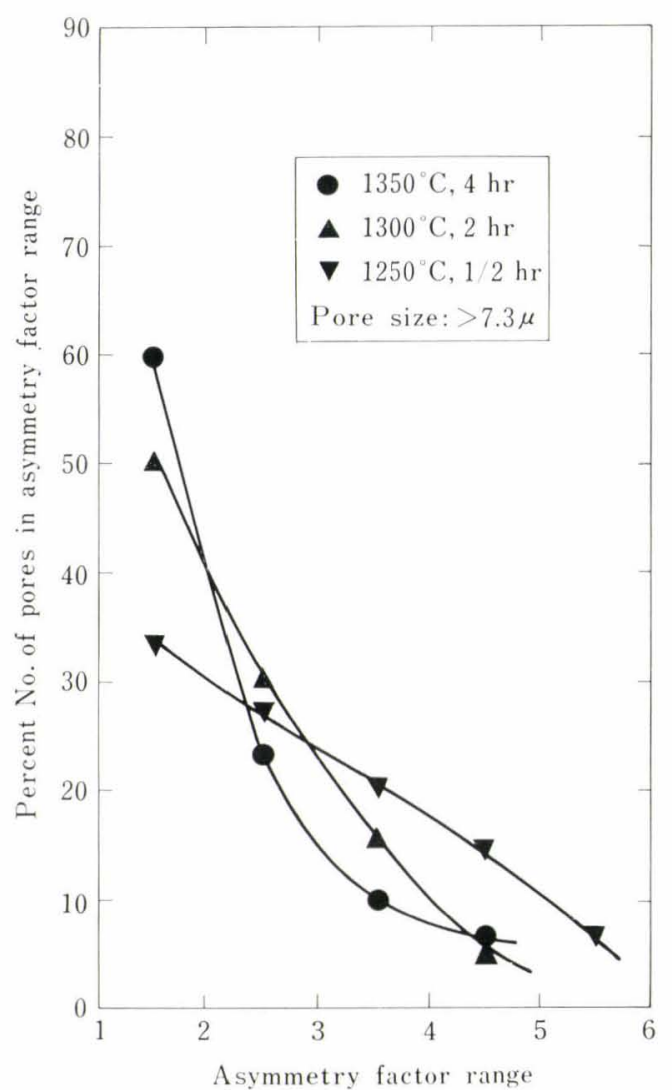

(c)

Table 3. Slopes of pore size distribution plots for sintered pellets

\begin{tabular}{c|ccc}
$\begin{array}{c}\text { Temp. } \\
\left({ }^{\circ} \mathrm{C}\right)\end{array}$ & \multicolumn{3}{|c}{ Slope } \\
\hline 1250 & 2.36 & $2 \mathrm{hr}$ & $4 \mathrm{hr}$ \\
\hline 1300 & 2.74 & 2.70 & 2.78 \\
1350 & 2.26 & 2.94 & 2.94 \\
& & 2.38 & 2.45
\end{tabular}

Shrinkage in large pores is negligible and in some cases growth is possible. The statistics of 100 pores for each sample is perhaps insufficient to show up the change in largest size pores, which though very small in number will however determine the limiting maximum size as well as the average size.

During the experimental steps of sintering and microstructure examination it was noted that large elongated pores having very small necks tend to break up into two or three smaller intermediate size pores at higher temperatures. The resulting distribution will depend on the balance between the shrinkage of very fine pores and the production of fresh intermediate size pores. However considering the precision of this kind of experiment the observed decrease in slope at $1350^{\circ} \mathrm{C}$ may only be apparent and may not be considered significant.

The average pore size increases with increasing degree of sintering as shown in Table 4. This is reasonable, because due to the increase in sintering fine pores are getting removed and the average will shift towards higher size. This is a very significant behaviour. 
Table 4. Average pore size of sintered pellets

\begin{tabular}{c|ccl}
\hline $\begin{array}{c}\text { Temp. } \\
\left({ }^{\circ} \mathrm{C}\right)\end{array}$ & \multicolumn{3}{|c}{ Average pore size $X($ /! $)$} \\
\hline 1250 & $1 / 2 \mathrm{hr}$ & $2 \mathrm{hr}$ & $4 \mathrm{hr}$ \\
\hline 1300 & 12.4 & 11.5 & 13.75 \\
1350 & 12.5 & 12.7 & 14.4 \\
& & 13.3 & 14.4
\end{tabular}

The porosity of the pellet will continue to decrease with increased sintering time because the total number of pores will continue to decrease. And yet at the same time the average pore size may increase due to a shift in distribution.

\section{Asymmetry Factor}

Percent number of pores have been plotted with respect to asymmetry factor range in Figs. 9 (p. 230) (a), (b), and (c) for different ranges of pore size to show the changes in shape of various pore size groups during the course of sintering. It was found that pores become more symmetrical by increasing the degree of sintering. This is reasonable because as the degree of sintering increases, pores shrink and try to become spherical. The symmetricity increases more rapidly in case of fine pores while the increase is very small in case of coarse pores. The symmetricity in coarse pores may also increase due to the disconnection of large pores into fine and more symmetrical ones.

The surface area of pores per unit volume of pellet has been calculated from porosity and pore size

$$
\begin{aligned}
& \text { Porosity } P=\frac{\text { Vol. of pores }}{\text { vol. of pellet }} \\
& \text { Surface area of pores/vol. of pellet } \\
& =\frac{\text { Surface area of pores }}{\text { vol. of pores }} \times \text { Porosity } \\
& =\frac{X^{2}}{\frac{X^{3}}{6}} \times P=\frac{6}{X} P .
\end{aligned}
$$

Surface area of pores $\left(\mathrm{cm}^{2} / \mathrm{g}\right)=\frac{6 P}{X d}$

where $X$ is the diameter of pores and $d$ is the bulk density of the material. It was observed that the surface area of pores per gramme of material varies from $284 \mathrm{~cm}^{2} / \mathrm{g}$ at $1 / 2 \mathrm{hr}$ to $270 \mathrm{~cm}^{2} / \mathrm{g}$ at $4 \mathrm{hr}$ for $1250^{\circ} \mathrm{C}$ and $260 \mathrm{~cm}^{2} / \mathrm{g}$ at $1 / 2 \mathrm{hr}$ to $153 \mathrm{~cm}^{2} / \mathrm{g}$ at $4 \mathrm{hr}$ for $1350^{\circ} \mathrm{C}$. The surface area of pores per gramme of material decreases with increasing the degree of sintering, which is reasonable because though the average pore size is increasing but the number of pores is decreasing, therefore the surface area of pores should decrease. The observations are in general agreement with an earlier work on the sintering of copper powder. ${ }^{17}$

Microstructures of various pellets sintered to different degrees have been shown in Photos. 1 (a), (b), (c), and (d). Here it can easily be seen that pores vary from a micron to $15-20 \mu$ in size. It can also be observed that for lower degree of sintering there are more fine pores while there are less fine pores in the pellets sintered to higher degree. Pores are generally present along the grain boundaries. Some regions with phase contrast are to be seen in both pure as well as doped hematite. This phase contrast may be due to the effect of orientation on some of the grains.

\section{Acknowledgements}

The authors wish to acknowledge the assistance rendered by Mr. B. Sharma, Mr. R. Prasad, Mr. V. N. Sharma and Mr. K. P. Mukerji of the Department of Metallurgical Engineering, in the experimental work.

\section{REFERENCES}

1) D. L. Johnson and I. B. Cutler: J. Amer. Ceram. Soc., 46 (1963), 541.

2) R. L. Coble: J. Amer. Ceram. Soc., 41 (1958), 55.

3) G. C. Kuczynski: Trans. AIME, 185 (1950), 169.

4) W. D. Kingery and M. Ber: J. Appl. Phys., 26 (1955), 1205.

5) R. L. Coble: J. Appl. Phys., 32 (1961), 787.

6) T. E. Clare: J. Amer. Ceram. Soc., 49 (1966), 159.

7) T. K. Gupta and R. L. Coble: J. Amer. Ceram. Soc., 51 (1968), 521

8) M.J. Banaister: Interdependence of Pore Removal and Grain Growth during Later Stages of Sintering in Berryllium Oxide, Sintering and Related Phenomena, Conference Proceedings, Notre Dame Univ., June 1965, Gordon and Breach, N. Y., 1967.

9) R. L. Fullman: Trans. AIME, 197 (1953), 447.

10) P. A. Beck, J. C. Kramer, L. G. Demer, and M. Holzworth: Trans. AIME, 175 (1948), 372.

11) B. Francois and W. D. Kingery:: The Sintering of Crystalline Oxides I. Interaction between grain boundaries and pores, Sintering and Related Phenomena, Conference Proceedings, Notre Dame Univ., June 1965, Gordon and Breach, N. Y., 1967.

12) W. D. Kingery and B. Francois: J. Amer. Ceram. Soc., 48 (1965), 546

13) S. N. Laha and A. R. Das: Isothermal Grain Growth and Sintering in Pure $\mathrm{ThO}_{2}$ and $\mathrm{ThO}_{2}-\mathrm{CaO}$ Compositions, To be pub., J. Nucl. Materials.

14) J. W. Cahn and R. L. Fullman: J. Metals, 8 (1956), 610.

15) E. F. Osborn and Arnulf Muan: Phase Equilibrium Diagrams of Oxide Systems. The System FeO- $\mathrm{Fe}_{2} \mathrm{O}_{3}-\mathrm{SiO}_{2}$, Published by American Ceramic Society, Columbus 14, Ohio, (1960).

16) L. Himmel, R. F. Mehl, and C. E. Birchenall: Trans. AIME, 197 (1953), 827.

17) F. N. Rhines, C. E. Birchenall, and L. A. Hughes: Trans, AIME, 188 (1950), 378. 